$3720 \mathrm{~cm}^{-1}$, however, shows a decrease in intensity which is not expected from the simple model.

It will be noted that the observed peaks are not equally spaced as they should be if the libration were harmonic or nearly so. Moreover, some of the peaks show a fine structure for which we have no interpretation. It is clear that the explanation of these details must await a refinement of the model.

Attempts were made to observe a fine structure in the Raman spectrum of single crystals, but only the fundamental was found. Efforts to observe the fundamental librational frequency in the far infrared, using $10 \mu$ thick sections, showed only a continuous absorption beginning near $700 \mathrm{~cm}^{-1}$ and extending beyond $250 \mathrm{~cm}^{-1}$, the lowest frequency which could be studied.

† This work was performed under the auspices of the U. S Atomic Energy Commission.

${ }^{1}$ W. R. Busing and H. A. Levy, J. Chem. Phys. 26, 563 (1957).

${ }^{2}$ R. T. Mara and G. B. B. M. Sutherland, J. Opt. Soc. Am. 43, 1100 (1953); 46, 464 (1956).

${ }^{3}$ H. E. Petch and H. D. Megaw, J. Opt. Soc. Am. 44, 744 (1954).

${ }^{4}$ R. M. Hexter and D. A. Dows, J. Chem. Phys. 25, 504 (1956).

\section{Uncertainties in the Measurement of the Oscillator Strength of the Ultraviolet Bands of $\mathrm{OH} \dagger$}

\author{
P. J. DyNe* \\ Guggenheim Jet Propulsion Center, California Institute \\ of Technology, Pasadena, California \\ (Received February 6, 1958)
}

$T$ HE $f$ value of the ${ }^{2 \Sigma}-{ }^{2} \Pi$ band system of $\mathrm{OH}$, measured by Oldenberg and Rieke ${ }^{1}$ is one of the few molecular oscillator strengths to have been measured. It is of importance in connection with the spectra of flames and other high temperature sources. In a redetermination of this $f$ value it was found that there were anomalous features in the relative intensities of the individual lines of the band system which cast some doubt on the validity of the technique used in both determinations.

In the present experiment ${ }^{2}$ which was essentially a duplicate of that performed by Oldenberg and Rieke, $\mathrm{OH}$ radicals were produced by thermal dissociation in a mixture of $\mathrm{O}_{2}$ and $\mathrm{H}_{2} \mathrm{O}$ vapor at atmospheric pressure. A mixture of these gases was passed through a Vycor absorption cell $50 \mathrm{~cm}$ long and heated to about $1100^{\circ} \mathrm{C}$ inside a furnace which was $100 \mathrm{~cm}$ long. This technique was an improvement over that of Oldenberg and Rieke in that the absorption path was confined to a region of relatively uniform temperature (temperature variations were $10^{\circ}-15^{\circ} \mathrm{C}$ along the length of the cell). Absorption lines of $\mathrm{OH}$ were photographed in the third order of a 21-ft grating spectrograph and their intensities measured by microphotometry. Although the resolving power of this instrument, as measured by the Rayleigh cri- terion, was not significantly different from that used in the earlier experiments, the absorption lines of $\mathrm{OH}$ were broader with a half-width of $0.86 \mathrm{~cm}^{-1}$ as against 0.56 $\mathrm{cm}^{-1} .^{1}$ The $f$ values obtained were about half those found by Oldenberg and Rieke. The measurements of the relative intensity of lines within the band showed some unexpected features.

The $f$ value of a spectral line is proportional to the integrated absorption coefficient $\int k_{\nu} d \nu=\int \ln \left(I_{\nu}{ }_{\nu}{ }^{0} I_{\nu}\right) d \nu$ where $I_{\nu}{ }^{0}$ is the incident light intensity and $I_{\nu}$ is the emergent light intensity. As is well known, a direct measurement of the $f$ value, using this relation, is only possible if the line is weak and broad compared with the effective slit width of the instrument. Using this technique it was found, both in the present work and that of Oldenberg and Rieke, that the relative intensities were not in agreement with theory; relative to the weak lines the stronger lines had only about half the predicted intensity. The results can be corrected by the semiempirical method of extrapolating to "zero intensity." In spite of the fact that the theoretical resolving power is about $0.15 \mathrm{~cm}^{-1}$ and that the half-width of the absorption lines was $0.6-0.9 \mathrm{~cm}^{-1}$ it appears that there was considerable loss of resolution and that the stronger lines were probably saturated.

The effect of self-absorption can be estimated by measuring the "total absorption" $\int\left[\left(I_{\nu}{ }^{0}-I_{\nu}\right) / I_{\nu}{ }^{0}\right] d \nu$ which is, in principle, independent of the resolving power. A plot of this quantity $v$ s log (relative strength of the line) for a series of absorption lines is termed a curve of growth. Absolute curves of growth, in which the abscissa is effectively log (absolute strength of line) have been calculated. ${ }^{3}$ Using these curves it is then possible to calculate a multiplicative factor in the logarithmic term which would make the measured total absorption lie on a true curve of growth. The line strength is then determined on an absolute scale.

The data of Oldenberg and Rieke can be recalculated in terms of the total absorption and a curve of growth plotted. Both these data and the newer data give a series of points which lie on a curve of growth for which the line shape parameter $a$ is between 0 and $0.5 \ddagger$ which means that the lines are almost purely Doppler broadened and show little pressure broadening. This would mean that the true line width is much less than that observed. It is, however, difficult to see why instruments with a resolving power of $0.15 \mathrm{~cm}^{-1}$ by the Rayleigh criterion should produce such broad lines. It is equally unexpected that there should be such a small amount of pressure broadening. In the author's opinion a likely explanation is that, in spite of the fact that the total absorption is independent of resolving power, the line strengths were not measured correctly and were consistently underestimated. The lines lie on a relatively flat portion of the curve of growth for values of $a$ of the order of unity or greater. The true $f$ values of the lines of $\mathrm{OH}$ may well be appreciably greater even than those obtained by Oldenberg and Rieke. 
I should like to express my thanks to Dr. R. M. Badger in whose laboratory the experimental work was carried out and to Dr. S. S. Penner for many stimulating discussions.

† Financial support for this work was provided by the Office of Naval Research under Contract Nonr-220(03), NR-015 210, and by the Daniel and Florence Guggenheim Jet Propulsion Center. The experimental work was carried out in the Gates and Crellin Laboratories of Chemistry.

* Post-Doctorate Research Fellow in Jet Propulsion, 1952-1953. Present address, Atomic Energy of Canada Limited, Chalk River, Ontario, Canada.

1 O. Oldenberg and F. F. Rieke, J. Chem. Phys. 6, 439 (1938).

${ }^{2}$ Guggenheim Jet Propulsion Center, Technical Report No. 12, Contract Nonr-220(03), NR 015 210, Pasadena (1954).

${ }^{3}$ W. Schutz, Z. Astrophys. 1, 300 (1930); E. F. M. van der Held, Z. Physik 70 (1930); S. S. Penner and R. W. Kavanagh, J. Opt. Soc. Am. 43, 385 (1953).

$\ddagger$

$$
a=\frac{\Delta \nu_{n}+\Delta \nu_{c}}{\Delta \nu_{D}}(\ln 2)^{k}
$$

where $\Delta \nu_{n}, \Delta \nu_{c}$, and $\Delta \nu_{D}$ are, respectively, the natural, collision, and Doppler half-widths. The use of one curve of growth for a number of different lines within a band assumes that all the lines are identically pressure broadened.

\section{Thermal Conductivity of Multicomponent Gas Mixtures*}

\author{
EDWARD A. Mason \\ Instilute for Molecular Physics, Universily of Maryland, \\ College Park, Maryland
}

(Received January 22, 1958)

$\mathrm{R}$ ECENT advances in the theory of heat conduction in gas mixtures now permit reliable calculations to be made for cases involving polyatomic, electronically excited, or chemically reacting molecules. ${ }^{1}$ The first step is the calculation of the coefficient of thermal conductivity for a mixture with all internal degrees of freedom imagined to be "frozen," so that the mixture behaves like a mixture of monatomic gases. Subsequent steps then correct for the effect of the internal degrees of freedom. The formulas used in the first step have been derived for multicomponent mixtures by Curtiss and Hirschfelder ${ }^{2,3}$ as an extension of the classical ChapmanCowling ${ }^{4}$ approach for binary mixtures. Unfortunately, these formulas involve extremely laborious numerical computation involving large determinants with complicated elements. ${ }^{5} \mathrm{~A}$ mixture of $n$ components requires the evaluation of a number of determinants of order $2 n$ and $(2 n+1)$, so that even for ternary mixtures the amount of labor is so excessive that simpler empirical formulas have been proposed. ${ }^{6}$ It is the purpose of this note to show how a much simpler formula can be derived with the same degree of rigor as the complicated Curtiss-Hirschfelder formula, and to show how this simpler formula can be reduced by suitable approximations to the same form as a successful empirical formula.

The complicated Curtiss-Hirschfelder formula can be thought of as being derived as a first approximation to the solution of an infinite set of simultaneous linear equations. ${ }^{7}$ The first approximation is obtained by truncating the set of equations and solving according to a variational theorem. A much simpler first approximation can be obtained by Kihara's method, ${ }^{8}$ in which one discards derivatives of a set of irreducible collision integrals. This procedure is exact for the Maxwellian intermolecular potential, and is a good first approximation for most other potentials. The off-diagonal elements of the set of equations become zero, and the following solution for the coefficient of thermal conductivity of an $n$-component mixture is easily obtained:

$\left[\lambda_{\mathrm{mix}}\right]_{1}=4\left|\begin{array}{cccc}L_{11} & \cdots & L_{1 n} & x_{1} \\ \cdot & & \cdot & \cdot \\ \cdot & & \cdot & \cdot \\ \cdot & & \cdot & \cdot \\ L_{n 1} & \cdots & L_{n n} & x_{n} \\ x_{1} & \cdots & x_{n} & 0\end{array}\right| /\left|\begin{array}{ccc}L_{11} & \cdots & L_{1 n} \\ \cdot & & \cdot \\ \cdot & & \cdot \\ \cdot & & \cdot \\ L_{n 1} & \cdots & L_{n n}\end{array}\right|$.

Here $x_{i}$ is the mole fraction of the $i$ th component, and the elements $L_{i j}$ can be written as

$$
\begin{aligned}
L_{i i}= & -\frac{4 x_{i}{ }^{2}}{\left[\lambda_{i}\right]_{1}}-\frac{16 T}{25 p} \sum_{k=1, k \neq i}^{n} \\
& \times \frac{x_{i} x_{k}\left[(15 / 2) M_{i}{ }^{2}+(5 / 2) M_{k}{ }^{2}+4 M_{i} M_{k} A_{i k}{ }^{*}\right]}{\left(M_{i}+M_{k}\right)^{2}\left[D_{i k}\right]_{1}} \\
L_{i j}(i \neq j)= & \frac{16 T}{25 p} \frac{x_{i} x_{j} M_{i} M_{j}\left(10-4 A_{i j}{ }^{*}\right)}{\left(M_{i}+M_{j}\right)^{2}\left[D_{i j}\right]_{1}}
\end{aligned}
$$

where $\left[\lambda_{i}\right]_{1}$ is the first approximation to the thermal conductivity coefficient of pure component $i$ in units of erg- $\mathrm{cm}^{-1}-\mathrm{sec}^{-1}-\mathrm{deg}^{-1}, p$ is the pressure in dyne $/ \mathrm{cm}^{2}$, $\left[D_{i k}\right]_{1}$ is the first approximation to the binary diffusion coefficient for components $i$ and $k$ in units of $\mathrm{cm}^{2} / \mathrm{sec}$, the $M$ 's are molecular weights, and the $A_{i k}{ }^{*}$ are di-

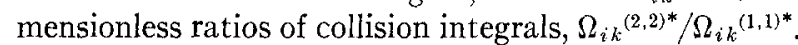
The $A_{i k}{ }^{*}$ depend on the temperature and the force law between molecules $i$ and $k$, and are generally of the order of unity. For the Lennard-Jones (12-6) and exp-six potentials, the $A_{i k}{ }^{*}$ are equal to about 1.1 over a large temperature range. Equations (1)-(3) can also be obtained from the Curtiss-Hirschfelder equations by setting the functions $B_{i k}{ }^{*}$ and $C_{i k}{ }^{*}$ in the latter equal to the Maxwellian model values of $5 / 4$ and $\frac{5}{6}$, respectively.

In Eq. (1) the off-diagonal elements $L_{i j}$ are much smaller than the diagonal elements $L_{i i}$, and are exactly zero if $A_{i j}{ }^{*}=\frac{5}{2}$. When the $A_{i j}{ }^{*}$ are all given this value, Eq. (1) becomes

$$
\begin{aligned}
{\left[\lambda_{\text {mix }}\right]_{1} } & =\sum_{i=1}^{n}\left[\lambda_{i}\right]_{1}\left[1+\sum_{k=1, k \neq i}^{n} G_{i k}\left(x_{k} / x_{i}\right)\right]^{-1}, \\
G_{i k} & =\frac{2}{5}\left(\frac{3 M_{i}+M_{k}}{M_{i}+M_{k}}\right) \frac{T\left[\lambda_{i}\right]_{1}}{p\left[D_{i k}\right]_{1}} .
\end{aligned}
$$

